\title{
Aplicación de las nuevas tecnologías en la rehabilitación del lesionado medular
}

\section{New technologies as a rehabilitation tool in spinal cord injury patients}

\section{Palabras clave}

Neurorrehabilitación, lesión medular, multidisciplinar, nuevas tecnologías, calidad.

\section{Keywords}

Neurorehabilitation, spinal cord injury, multidisciplinary, new technologies, quality.
“El término 'neurorrehabilitación' es entendido como el proceso destinado a reducir la deficiencia, la limitación de la actividad y la restricción de la participación que padecen las personas como consecuencia de una enfermedad neurológica, y en el que los profesionales involucrados en dicho ámbito tendrán como objetivo la reducción del grado de afectación funcional del paciente".

CANo de la Cuerda (20I6: 79-84).

\section{Introducción}

La lesión de médula espinal es una alteración en la médula espinal que puede producir una pérdida, total o parcial, de sensibilidad y/o de movilidad voluntaria por debajo de la región afectada, además de incontinencia, trastornos sexuales y otros de origen vegetativo (Institut Guttman, s. f.) pudiendo ser causada por un traumatismo, una enfermedad o tener un origen congénito (Zárate-Kalfópulos et al., 2016: IOI6-IOI9). Las consecuencias de la lesión medular son irreversibles, por lo tanto, el tratamiento neurorrehabilitador de una persona con lesión medular se prevé que pueda durar toda la vida.

El éxito en la neurorrehabilitación de una persona con lesión medular depende de muchos factores pero

\section{Sara Cáceres Saavedra}

<rehabilitacion@medular.org>

Coordinadora de Rehabilitación del departamento de Rehabilitación en la Fundación del Lesionado Medular

María Belén Gómez Saldaña <terapiaocupacional@medular.org>

Coordinadora de Terapia Ocupacional del departamento de Rehabilitación en la Fundación del Lesionado Medular

\section{María Jesús García Carpintero}

Terapeuta Ocupacional del departamento de Rehabilitación en la Fundación del Lesionado Medular

\section{Marina Milian Alonso}

Fisioterapeuta del departamento de Rehabilitación en la Fundación del Lesionado Medular

\section{Ana Arroyo Arias}

<fisioterapia@medular.org>

Coordinadora de Fisioterapia del departamento de Rehabilitación en la Fundación del Lesionado Medular

\section{Lía Cascante Gutiérrez}

Fisioterapeuta del departamento de Rehabilitación en la Fundación del Lesionado Medular

\section{Para citar:}

Cáceres, S. et al. (20I7): "Aplicación de las nuevas tecnologías en la rehabilitación del lesionado medular". Revista Española de Discapacidad, 5 (I): 229-236.

Doi: <https://doi.org/IO.5569/23405 IO4.05.OI.I3> 
es indiscutible que un trabajo en equipo multidisciplinar es uno de los condicionantes que predisponen a su logro. Dada la variabilidad de la sintomatología dentro de un mismo diagnóstico global, y para plantear un tratamiento personalizado, cobra gran importancia la valoración (Maggioni et al., 2016: 72) inicial de la persona que acude a rehabilitación. Y, puesto que la lesión puede provocar gran discapacidad, la valoración conjunta de todo el equipo es necesaria (Vasilchenko et al., 2016), abordando desde aspectos psicosocioeconómicos y del entorno de la persona, a cuestiones médicas y farmacológicas, información y formación de ciertas pautas diarias a seguir y de señales de alarma a detectar, así como formación y acondicionamiento físico y asesoramiento en productos de apoyo necesarios para conseguir dos objetivos claves, como son mantener y/o mejorar la calidad de vida, y fomentar la máxima independencia posible (Lo et al., 20I6: I958I968).

En este largo caminar durante su rehabilitación, los objetivos de la persona variarán según la fase en la que se encuentre. Dada la forma abrupta en la que aparece la lesión medular, los objetivos iniciales van fundamentalmente encaminados a orientar, informar y asesorar a la persona en todo lo necesario sobre su lesión y los comportamientos a seguir para prevenir complicaciones secundarias (Stillman et al., 20I7). También es en un primer momento cuando se realiza un trabajo de fortalecimiento físico, de conocimiento y adaptación al nuevo esquema corporal, de reeducación funcional para las actividades de la vida diaria, de acondicionamiento del entorno para facilitar la accesibilidad y la autonomía, así como lograr la reintegración a su entorno social habitual. Sin embargo, una vez superados estos objetivos, la dificultad de un tratamiento tan a largo plazo es la de combatir la rutina e invisibilidad de los beneficios de un tratamiento de mantenimiento, por lo que nuestro mayor esfuerzo va a ir encaminado a reducirlas en la medida de lo posible y a mantener la motivación y el interés en su autocuidado y prevenir las posibles complicaciones secundarias a las consecuencias de la lesión. Una de las herramientas con las que contamos para ello son las nuevas tecnologías en neurorrehabilitación, siendo posible adaptarlas para el tratamiento en lesionados medulares.

Las nuevas tecnologías se han ido introduciendo como una herramienta más de neurorehabilitación durante los últimos años (Calabró et al., 2016: 503-I4). Gracias a los últimos desarrollos tecnológicos y sus diferentes aplicaciones, contamos con diferentes dispositivos que facilitan la diversidad de actividades como complemento al tratamiento rehabilitador tradicional (Fasoli et al., 2003: 477-482; Fazekas et al., 2016:I48-I 54). En este artículo queríamos hacer un breve repaso de ellos.

\section{Nuevas tecnologías en neurorrehabilitación dirigidas a personas con lesión medular}

A. Dispositivos robóticos: los sistemas de control de estos dispositivos, además de asistir los movimientos, permiten obtener medidas fiables, objetivar la valoración (Maggioni et al., 2016: 72) y la evolución. El tratamiento puede ser aplicado sin la supervisión constante del personal de rehabilitación (Hesse et al, 2003: 7057IO). Cabe destacar entre sus beneficios la capacidad de disminuir la fatiga de paciente y terapeuta, lo que permite aumentar la intensidad de las sesiones y el número de repeticiones (Hilderley et al., 20I6: I886). También mejora la precisión del control motor gracias, entre otras cosas, al feedback que recibe el paciente, aportando seguridad y calidad en el movimiento. Otra de las ventajas es poder registrar y documentar de forma objetiva el progreso del usuario. Entre los inconvenientes más destacados se encuentra la complicada colocación de los sistemas, lo que requiere de un mayor tiempo invertido. Y en algunos de ellos se evidencia la necesidad de un diseño que permita un desarrollo más fisiológico. Para tratar la lesión medular podemos destacar: 
a. En miembros superiores: dispositivos cuyas modalidades de trabajo son bilaterales, pudiendo ser adaptados a las características de cada paciente y recoger información objetiva (Hussain et al., 2016: 477) que permite salvar la subjetividad interprofesional. Sus mayores inconvenientes radican en que los movimientos no son funcionales y en la dificultad de adaptarlos para todos los usuarios en sillas de ruedas eléctricas:

i. Dispositivos que trabajan analíticamente la flexo-extensión de muñeca y prono-supinación del antebrazo, bien en modo pasivo, activo o resistido, con los objetivos de mantener y/o mejorar balances articular y muscular (Fasoli et al., 2003: 477-482).

ii. Dispositivos que realizan una movilización pasiva de las articulaciones metacarpofalángicas e interfalángicas de los cuatro dedos -a excepción del pulgarteniendo la opción de añadir vibración como estímulo, lo que favorece la disminución de la espasticidad, la prevención de retracciones articulares y proporciona un importante estímulo sensoriomotor a la mano.

b. En miembros inferiores: destacan los dispositivos cuyo objetivo es iniciar una marcha precoz -simulada o real-, además de estimular el sistema cardiorespiratorio (Han et al., 20I6) con ejercicio aeróbico y anaeróbico, aportar beneficios a nivel del sistema músculoesquelético, del retorno venoso y de tránsito intestinal. De gran interés es el registro histórico que guarda de todas las sesiones y que puede ser extraído para el tratamiento de los datos. Entre los inconvenientes a destacar, encontramos el tiempo de instalación requerido, como ya se ha citado anteriormente, y la incomodidad del arnés que limita movimientos y señala un número importante de usuarios.

i. Aquellos que simulan la marcha sin desplazamiento aportan, simultáneamente a la bipedestación por plano inclinado, una movilización de los miembros inferiores, pasiva o activa-asistida. Cuentan con la ventaja de poder ser usados también con personas con una lesión medular cervical.

ii. Otros que facilitan una marcha precoz permiten suspender parcialmente el peso para aquellos pacientes que no toleren la carga completa. Constan de un tapiz rodante combinado con una plataforma de presión que recoge información durante la sesión sobre el tiempo que ha permanecido la carga en cada pie y la regularidad y longitud del paso que ha llevado. Es posible, además, inclinar el tapiz para entrenar la marcha con pendiente, cambiar la dirección del tapiz hacia delante y hacia detrás, incluso provocar cambios bruscos de dirección en los cuatro ejes, para entrenar los reflejos ante imprevistos que pueden surgir al caminar en el exterior. También se puede añadir, si es necesario, un feedback auditivo para estimular la marcha regular. Todo ello con el objetivo de aumentar el perímetro de marcha y la resistencia al esfuerzo.

B. Exoesqueletos: entre los dispositivos que buscan un movimiento más fisiológico $\mathrm{y}$ funcional podemos subrayar los exoesqueletos tanto de miembros inferiores como superiores. Estos sistemas utilizan un esqueleto externo que:

a. En miembros superiores (KlamrothMarganska et al, 20I4: I 59-I66): permite a pacientes, a través de ejercicios de realidad virtual semi- 
inmersiva, la práctica simulada de actividades funcionales, proporcionando una valoración objetiva del paciente y posibilitando estudios longitudinales intra e interpersonales.

i. Desgravita lo necesario en el miembro a trabajar.

ii. Permite adaptar los ejercicios a las capacidades del usuario.

b. En miembros inferiores: posibilita realizar la marcha de una manera similar a la natural (Louie et al, 2OI 5: I 2-82) y permite la recogida de estadísticas para su posterior uso.

i. Dispone de diversos ajustes pudiendo seleccionar grados de fuerza ejercida por el dispositivo en función de la que tenga la persona, y tipos de acción, de modo que pueda ser activado por el propio paciente a medida que avanza en el proceso rehabilitador, siempre bajo supervisión de personal cualificado. El exoesqueleto facilita una rehabilitación de la marcha funcional y segura (Yang et al., 2016: I I4-II9), (Bronnikov et al., 20I6: 30-34). Ayuda a caminar desde la primera sesión como también, según las posibilidades de cada persona, a conseguir un patrón de marcha próximo al natural (Krishnan et al., 2016: 78I-786).

ii. Además, incorpora unos sensores de carga que activa el propio paciente, según transfiera la carga hacia un miembro, u otro, para permitir dar el paso.

iii. Por otro lado, aporta un feedback auditivo que informa, tanto al paciente como al fisioterapeuta, cuando la transferencia de la carga es la adecuada, según el valor previamente establecido.
C. Realidad virtual: los profesionales en neurorehabilitación cuentan además con otra herramienta, la realidad virtual, que trata de simular el ambiente de la vida real mediante un software informático con el que el usuario puede experimentar a través de un interfaz ser humano-máquina. Puede ser inmersiva, semi-inmersiva o no inmersiva. Gracias al feedback (Roosink et al., 2016: 227-235) que recibe el usuario, éste es capaz de corregir sus movimientos de forma autónoma (Ferreira dos Santos et al., 2016: I44). Se debe añadir por otro lado un beneficio extra como es el aspecto lúdico que aporta el hecho de interaccionar a través de videojuegos. De esta forma se reduce la sensación rutinaria, a la vez que aumenta la percepción de mejorar su entrenamiento físico $(60,38 \%)$ y su motivación (73,59\%) (Pérez Seco et al., 20I4: 20I3-7087). Entre las herramientas más destacadas en lesión medular se encuentran los guantes robóticos y las consolas de nueva generación:

a. Guantes robóticos (realidad virtual semi-inmersiva): que permiten una valoración y tratamiento de la mano analíticos y objetivos (DimbwadyoTerrer et al., 2016: 462-467).

i. El sistema está compuesto por un dispositivo ergonómico (guante) y un software de soporte.

ii. Ofrece varios juegos digitales, específicamente diseñados para diversos objetivos.

iii. Guían al paciente en el proceso de rehabilitación haciéndolo más ameno, además de añadir un grado de motivación necesaria.

b. Consolas de nueva generación (realidad virtual no inmersiva): su bajo coste hace que dinamizar las terapias esté al alcance de todos y su uso cada vez esté más extendido. Existen diferentes tipos de consolas:

i. Sin uso de mando: el usuario es el propio mando. Las partes del cuerpo se convierten en 
un esqueleto en movimiento. Éstas requieren calibración con un movimiento concreto, poder realizar el gesto de saludo y realizar movimientos más complejos que con otras consolas.

ii. Con mando inalámbrico: existen diferentes adaptaciones para poder permitir a usuarios de diferente nivel de funcionalidad participar en los juegos. Permite trabajar diferentes objetivos, tales como la coordinación y la destreza de la mano (Diez-Alegre y Cano de la Cuerda, 201 2: 23-30), entre otros, en función de la necesidad de cada persona y mediante la selección del juego apropiado.

iii. Sensores de movimiento en USB: micro-cámaras incorporadas en su interior que captan los movimientos de las manos y los traslada a la pantalla de un ordenador en un contexto tridimensional. Puede utilizarse para trabajar diferentes habilidades manipulativas: pinzas, flexoextensión de muñeca, rapidez de movimientos, escritura, etc.

D. El sistema de entrenamiento de agudeza visual dinámica también se puede utilizar en lesión medular, siendo útil para entrenar la respuesta refleja y óculomotora (Wells et al., 20I4: I45-I 50) a los numerosos estímulos de este tipo existentes en la vida real, y acompañarlo con un entrenamiento para el control de tronco (Vesia et al., 2008: I4-20) y/o el equilibrio tanto en posición sedente como en bipedestación, según las posibilidades de cada paciente. Este dispositivo consiste en una pantalla vertical de luces que forman cinco anillos concéntricos conectados a un software que permite guardar los datos de cada usuario, objetivando los resultados de velocidad y el número de aciertos en cada sesión.
E. La valoración a través de la plataforma de medición de presiones ha demostrado una eficacia objetivable para la prevención de úlceras por presión y un adecuado posicionamiento en silla de ruedas. Consiste en un dispositivo con forma de manta con sensores unidos a un software, lo que ayuda a identificar aquellas zonas donde la presión supera los criterios recomendados. Este dispositivo ofrece una imagen de un mapa de presiones donde aparecen las zonas de riesgo por elevada presión, proporcionando un feedback visual a terapeuta y paciente. Es una herramienta esencial en el adecuado posicionamiento de personas con lesión medular, proporcionando datos objetivos que dan al terapeuta y al usuario la información necesaria para poder conseguir el posicionamiento más adecuado y seguro. Este posicionamiento se consigue con una buena elección y ajuste de la silla de ruedas (manual o eléctrica), con la elección correcta tanto del cojín (Gil-Agudo et al., 2016: 227-35) como del respaldo en los casos en los que sea necesario, recomendaciones posturales y corrección de malos hábitos del paciente, así como de los complementos posicionales que sean necesarios. No hay una solución universal, sino que las soluciones son tan variadas como usuarios con lesión medular, y estas soluciones a veces tienen un elevado coste (Soldevilla Agreda et al., 2008: 275-297). De ahí la importancia de una buena valoración inicial del paciente y de herramientas como la plataforma de medición de presiones que colaboran en la prevención de las úlceras por presión (Hamanami et al., 2004: 37-44) y proporcionan un buen posicionamiento asegurando la máxima calidad de vida y autonomía de las personas con lesión medular.

\section{Conclusiones}

Si bien las nuevas tecnologías aportan oxigenación a los tratamientos a largo plazo 
Sara Cáceres Saavedra • María Belén Gómez Saldaña • María Jesús García Carpintero •

y los usuarios reciben con satisfacción la utilización de las mismas durante las sesiones de rehabilitación, su utilidad beneficiosa no sería posible sin el equipo humano que evalúa, sigue y orienta en todo el proceso a los pacientes.
Son, por tanto, una buena herramienta de la que disponer para conseguir objetivos concretos (Waldner et al., 2009: I73-I77), siempre que un experto sea quien las elija y manipule. 
Referencias bibliográficas

Bronnikov, V. A. et al. (2016): «Evaluation of motor skills recovery in post-stroke patients in the process of complex rehabilitation with the use of robotic kinesiotherapy". Zh Nevrol Psikhiatr Im S S Korsakova, I I6 (9): 30-34.

Calabrò, R. S. et al. (20I6): "Robotic gait rehabilitation and substitution devices in neurological disorders: where are we now?". Neurol Sci, 37 (4): 503-5I4.

Cano de la Cuerda, R. (20I6): "Frenkel: ¿un precursor de la neurorrehabilitación?”. Revista de neurología, 63 (2): 79-84.

Diez-Alegre, M. I. y Cano de la Cuerda, R. (20I2): "Empleo de un video juego como herramienta terapéutica en adultos con parálisis cerebral tipo tetraparesia espástica. Estudio piloto”. Fisioterapia, 34 (I): 23-30.

Dimbwadyo-Terrer, I. et al. (2016): “Upper limb rehabilitation after spinal cord injury: a treatment based on a data glove and an immersive virtual reality environment”. Disabil Rehabil Assist Technol, I I (6): 462-467.

Fasoli, S. E. et al. (2003): "Effects of robotic therapy on motor impairment and recovery in chronic stroke". Arch Phys Med Rehabil, 84: 477-482.

Fazekas, G.et al. (20I6): "New opportunities in neuro-rehabilitation: robot mediated therapy in conditions post central nervous system impairments". Ideggyogy Sz, 69 (5-6): I 48-I 54.

Ferreira dos Santos, L. et al. (20I6): "Movement visualisation in virtual reality rehabilitation of the lower limb: a systematic review". Biomed Eng Online, I 5 (Suppl 3): I 44.

Gil-Agudo, A. et al. (20I6): "Interactive virtual feedback improves gait motor imagery after spinal cord injury: An exploratory study". Restor Neurol Neurosci, 34 (2): 227-235.

Hamanami, K. et al. (2004): "Finding the optimal setting of inflated air pressure for a multi-cell air cushion for wheelchair patients with spinal cord injury”. Acta Med Okayama, 58 (I): 3744 .

Han, E. Y. et al. (2016): "Robot-assisted gait training improves brachial-ankle pulse wave velocity and peak aerobic capacity in subacute stroke patients with totally dependent ambulation: Randomized controlled trial”. Medicine (Baltimore), 95 (4I): e5078.

Hesse, S. et al. (2003): "Upper and lower extremity robotic devices for rehabilitation and for studying motor control”. Curr Opin Neurol, (6): 705-710.

Hilderley, A. J. et al. (2016): “Comparison of a robotic-assisted gait training program with a program of functional gait training for children with cerebral palsy: design and methods of a two group randomized controlled cross-over trial”. Springerplus, 5 (I): I 886.

Hussain, A. et al. (2016): "Self-Paced Reaching after Stroke: A Quantitative Assessment of Longitudinal and Directional Sensitivity Using the H-Man Planar Robot for Upper Limb Neurorehabilitation". Front Neuroscience, Iо: 477 .

Institut Guttman (s. f.): Lesión medular (en línea). $<$ http://www.guttmann.com/es/treatment/lesionmedular>, acceso 17 de febrero de 2017.

Klamroth-Marganska, V. et al. (20I4): “Threedimensional, task-specific robot therapy of the arm after stroke: a multicentre, parallel-group randomised trial". Lancet Neurol, I3 (2): I 59I 66.

Krishnan, V. et al. (2016): "Robotic-assisted locomotor training enhances ankle performance in adults with incomplete spinal cord injury". J Rehabil Med, 48 (9): 78I-786.

Lo, C. et al. (2016): "Functional Priorities in Persons with Spinal Cord Injury: Using Discrete Choice Experiments To Determine Preferences". J Neurotrauma, 33 (2I): 1958 I968. 
Louie, D. R et al. (201 5): "Gait speed using powered robotic exoskeleton after spinal cord injury: a systematic review and correlational study". J Neuroeng Rehabil, I2: 82.

Maggioni, S. et al. (2016): "Robot-aided assessment of lower extremity functions: a review". J Neuroeng Rehabil, I3 (I): 72.

Pérez Seco, M. et al. (20I4): "Virtual Reality in Rehabilitation". Journal of Accessibility and Design for All, 4 (3): 223-237.

Roosink, M. et al. (2016): "Interactive virtual feedback improves gait motor imagery after spinal cord injury: An exploratory study". Restor Neurol Neurosc, 34 (2): 227-235.

Soldevilla, A. et al. (2008): "Impacto social y económico de las úlceras por presión. Enfermería y Úlceras por presión: De la Reflexión sobre la Disciplina a las Evidencias en los Cuidados". Grupo ICE - Investigação Cientifica em Enfermagem, 275-297.

Stillman, M. D. et al. (20I7): "Complications of Spinal Cord Injury Over the First Year After Discharge from Inpatient Rehabilitation". Arch Phys Med Rehabil.
Vasilchenko, E. et al. (2016): “A descriptive study on the functioning profile of patients with spinal cord injury in a rehabilitation center in Russia”. Spinal Cord, 55 (5): 489-496.

Vesia, M. et al. (2008): "Correlations of selected psychomotor and visuomotor tests with initial dynavision performance". Perceptual and Motor Skills, IO7 (I): I4-20.

Waldner, A. et al. (2009): "Transfer of scientific concepts to clinical practice: recent robotassisted training studies". Funct Neurol, 24 (4): I73-I 77

Wells, A. J. et al. (2OI4): "Reliability of the Dynavision $^{\mathrm{TM}} \mathrm{D}_{2}$ for Assessing Reaction Time Performance". J Sports Sci Med, I3 (I): I 45 -I 5 O.

Yang, H. E. et al. (20I6): «Structural and functional improvements due to robot-assisted gait training in the stroke-injured brain". Neuroscience letters, 637: II4-II9.

Zárate-Kalfópulos, B. et al. (2016): “Demographic and clinical characteristics of patients with spinal cord injury: a single hospital-based study". Spinal Cord, 54 (I I): Ior6-Iorg. 